\title{
Clase y voto: intereses, identidades y preferencias
}

\author{
Ludolfo Paramio
}

Instituto de Estudios Sociales Avanzados (CSIC)

\begin{abstract}
Se discute en primer lugar el uso ambiguo y contradictorio del concepto de divisoria social (cleavage) para analizar la relación entre clase y voto, y se propone después estudiar esta relación en un marco en el que los intereses económicos, definidos por la estructura social, son sólo un punto de partida. Las identidades sociales y políticas de los electores, en este esquema, juegan un papel decisivo para determinar las preferencias de los electores, lo que implica que las explicaciones económicas del voto subestiman los factores propiamente políticos.
\end{abstract}

As a tool to explain electoral behaviour, the concept of class cleavage is often flawed by the equivocal and contradictory use of the term. Economic interests, as defined by the social class structure, offer an alternative point of departure. But social and political identities play a crucial role in the definition of voters' preferences, and therefore economic theories of voting underrate the weight of political factors in electoral behaviour.

\section{Introducción}

En los estudios sobre clase y voto se suele partir de la clase como una de las líneas divisorias fundamentales (cleavages) de nuestras sociedades, y siguiendo una perspectiva clásica se presupone que debe explicar en gran medida el comportamiento electoral. O, por el contrario, se intenta mostrar que no es así, y que dicha perspectiva clásica ya no es válida. Se puede argumentar que este planteamiento inicial es profundamente equívoco, dado el uso polisémico que damos al término cleavage. Una posibilidad más sensata sería entonces tratar de ver en qué medida la estructura de clase condiciona los intereses de los electores.

Pero la idea de que los intereses determinan las preferencias de los electores implica que éstas son unidimensionales, lo que encaja mal con la perspectiva más reciente sobre los valores en las sociedades desarrolladas. Independientemente de que se acepten o no los planteamientos de Inglehart sobre el ascenso de nuevos valores posmaterialistas, existe una amplia evidencia de que los electores interpretan la misma evolución de la economía en función de parámetros que no se reducen a los propios intereses materiales, sino que incluyen criterios políticos y sociales. Por otra parte, en el análisis de los resultados de las campañas surgen razones para pensar que los electores deciden sobre cuestiones concretas, a partir de su valoración previa respecto a ellas.

La perspectiva que cabe adoptar es dual: aceptar que la clase determina los intereses materiales y estudiar después cómo las identidades sociales y políticas crean el marco de interpretación de tales intereses y las metapreferencias a partir de las cuales algunas cuestiones se convierten en cruciales a la hora de decidir el sentido del voto. En este marco se pueden analizar los efectos de los discursos y estrategias partidarias sobre la autoidentificación de unos electores cuya posición de clase les condiciona en términos de 
intereses materiales.

\section{1. ¿De qué hablamos cuando hablamos de cleavages?}

El término cleavage, que podríamos traducir por línea divisoria, es uno de los más usados en sociología electoral. Refiriéndose al trabajo clásico de Lipset y Rokkan (1967), se discute en qué medida las grandes divisorias sociales, de clase, religión, etnia y cultura (nacional), surgidas en Europa como líneas de fractura en los procesos de formación del Estado nacional, de industrialización y de ampliación de la democracia, permanecerían congeladas y constituirían aún la estructura condicionante del comportamiento electoral. Esta idea ha venido siendo contrastada por diversos autores con resultados un tanto previsibles: el peso de estas divisorias a la hora de explicar el comportamiento electoral ha venido disminuyendo, y tiende a seguirlo haciendo (Franklin, 1992), pero sin embargo aún sigue siendo suficientemente significativo como para considerar válida la tesis (Bartolini y Mair, 1990).

La previsibilidad de tales resultados es consecuencia de la polisemia del término. En efecto, al hablar de cleavages para explicar el voto podemos referirnos a tres hechos bien distintos: (1) correlación entre posición estructural y voto; (2) correlación entre posición estructural e identificación política; (3) coincidencia entre las divisorias sociales y las líneas de confrontación electoral. Tomando el caso de la clase social, al que me ceñiré en lo sucesivo, tendríamos: (1) correlación entre posición de clase y voto; (2) correlación entre posición de clase e identificación con partidos de clase; (3) definición de la confrontación electoral en torno a la oposición entre intereses de clase.

La tercera posibilidad es muy infrecuente hoy en día: si un partido convierte la divisoria de clase en eje de su programa o de su campaña electoral renuncia, automáticamente, al apoyo de segmentos del electorado que podrían beneficiarse del contenido objetivo de sus propuestas programáticas, pero se sentirán simbólicamente rechazados e incluso amenazados en su propia identidad social. (Esto no sólo es cierto para los partidos obreros, sino mucho más para los partidos conservadores: durante los años 80, las políticas más agresivas a favor de los intereses del capital financiero y del gran empresariado se han presentado como defensa de los intereses del ciudadano de a pie frente al Estado depredador.) En este sentido, la activación política de las divisorias de clase sólo puede esperarse en situaciones defensivas, para consolidar el núcleo duro de un electorado desmovilizado, o como un submensaje focalizado dentro de una campaña global dirigida al conjunto del electorado.

Sin embargo, éste es el punto de arranque en el proceso histórico que da sentido al razonamiento de Lipset y Rokkan: la confrontación política (en la fase de extensión de los derechos políticos) se habría centrado en las divisorias de clase, y de esa confrontación habrían surgido identidades políticas fuertes, los partidos obreros frente a los conservadores, que marcarían hasta hoy la política europea y configurarían los sistemas de partidos. Pero habría que aceptar en todo caso que la discusión sobre la pertinencia explicativa actual de las divisorias de clase debe circunscribirse a las otras dos posibilidades: la correlación entre posición de clase y voto o entre posición de clase e identificación partidaria.

En el caso de la identificación partidaria, tenemos dos tipos de problemas. El primero y más grave surge de la existencia de diversas identidades políticas que aparecen en principio como correspondientes a un mismo espacio de clase. La coexistencia en Europa, desde 1921, de partidos socialdemócratas (en un sentido amplio del término) y partidos comunistas, no puede explicarse ex ante por la existencia de diferencias de clase en sus apoyos o electorados, aunque quepa encontrar en su desarrollo posterior un mayor o 
menor arraigo en diferentes segmentos de clase. Por tanto es preciso reconocer que la posición de clase, todo lo más, configura un amplio espacio político dentro del cual pueden competir identidades políticas distintas.

Ahora bien, esta indeterminación de la identificación política a partir de las divisorias sociales no es precisamente un fenómeno secundario. En su contrastación en la Alemania de Weimar de la tesis de Converse sobre la identificación partidaria, Shively (1972) llegaba a la conclusión de que la transferencia de votos a los nazis se había producido dentro de un mismo espacio político (el de los partidos protestantes no marxistas) sin afectar sustancialmente a la distribución global en espacios del electorado. Evidentemente, esta recomposición del peso de las identidades políticas dentro de una misma divisoria religioso-ideológica habría tenido consecuencias dramáticas, pero podríamos pensar que demuestra la distinta pertinencia explicativa de las estructuras de clase frente a las religiosas y culturales.

Examinando los datos de Shively, sin embargo, ese aparente consuelo se disipa: entre 1932 y 1933 entre 6 y 7 puntos de porcentaje del voto pasaron del espacio (bloque, en expresión de Shively) marxista al de los partidos protestantes no marxistas. Si atribuimos a la frontera entre marxistas y no marxistas un carácter de clase debemos admitir que no era una frontera para nada impermeable, y si la consideramos puramente ideológica debemos concluir que la divisoria de clase no es especialmente significativa en un proceso político de tan radical impacto como el ascenso electoral del nacional-socialismo. (Caben hipótesis mixtas, pero no mejoran la situación, sino que debilitan aún más el valor explicativo de ambos tipos de divisoria.)

Existe un segundo problema en lo que se refiere a la correlación entre divisorias de clase e identidades partidarias: el de su cambiante peso e intensidad a lo largo del tiempo. Sobre esta cuestión se han realizado numerosos estudios, y por una parte existe un cierto consenso sobre la erosión de los alineamientos partidarios, en un doble sentido: serían menos los electores identificados con un partido y su identificación constituiría un vínculo más débil que el supuestamente existente en los años de la posguerra (Abramson, 1976 y 1992). Lo más significativo, sin embargo, es que el número de electores no alineados varía en función de acontecimientos coyunturales, y no sólo tendencialmente ni ante situaciones excepcionales (Clarke y Suzuki, 1993).

En efecto, parece evidente que existen situaciones de crisis social (choques políticos o económicos) que afectan a la identificación partidaria, y en ellas se producen las elecciones críticas (Key, 1955) que redefinen los alineamientos partidarios para un período prolongado, no necesariamente produciendo conversiones, pero sí desalineamientos: la desilusión de unos votantes y la nueva identificación de otros provocaría el realineamiento global del electorado (Leithner, 1997). La misma posibilidad de estos realineamientos se puede entender como una muestra de la pérdida de peso de las divisorias sociales en cuanto determinantes de la conducta electoral (Burnham, 1970), en su aspecto de identificación política.

Aun admitiendo que las identificaciones partidarias parecen mostrar una muy notable estabilidad (Schickler y Green, 1997; Green y Palmquist, 1994), resulta patente que hoy son más débiles y que no cierran la posibilidad de cambios electorales espectaculares. Pero si además tal identificación oscila en función de circunstancias coyunturales, como muestran Clarke y Suzuki, y no sólo en elecciones críticas, parece lógico admitir que el papel de las divisorias sociales en la determinación de la identificación política es bastante más reducido de lo que se suele entender, y que es más realista una teoría como la de Converse (1969) que pretende explicar tal identificación en función de la socialización y el aprendizaje. 
Nos queda entonces la acepción más elemental de cleavage: la existencia de una correlación entre el voto y la estructura social definida por las divisorias sociales (en este caso por la posición de clase). Como es bien sabido, existe un número considerable de estudios que pretenden mostrar la pérdida de significado de la clase social para predecir o explicar el comportamiento electoral. Ahora bien, tras revisar una gran parte de ella, lo más que se puede afirmar es que esta tesis es claramente exagerada y carece del necesario apoyo en los datos. Considerando el período posterior a la segunda guerra mundial, "en ningún país capitalista democrático el voto ha llegado a ser completamente independiente de la clase social en unas elecciones nacionales" (Manza, Hout y Brooks, 1995: 158).

Es difícil no aceptar una afirmación tan cauta. En efecto, sería realmente sorprendente que el voto llegara a ser completamente independiente de la clase social, si suponemos que la definición de la posición de clase tiene alguna pertinencia sociológica. Con cualquier definición que utilicemos (ya sea en términos de renta, de estatus, de oportunidades en el mercado o de posición en las relaciones de producción) cabe imaginar que obtendremos un mapa de intereses sociales que en buena lógica mostrará alguna correlación con el comportamiento electoral. No es fácil imaginar una confrontación electoral en la que las distintas opciones de política no tengan implicaciones específicas para los intereses de los colectivos definidos en términos de clase.

Llegados aquí, sin embargo, parece evidente que hablar de una divisoria social es equívoco. Lo que tenemos es una estructura social que define intereses, y cabe suponer que tales intereses tienen un papel importante a la hora de determinar las preferencias de los electores. Ahora bien, si en la confrontación electoral ningún partido pretende activar políticamente las divisorias de clase, ni la clase determina en un sentido fuerte las identificaciones partidarias, el concepto de cleavage nada ofrece a la sociología electoral, fuera del curioso ejercicio de tratar de comprobar de vez en cuando si la tesis de la congelación de las divisorias sociales sigue siendo válida. Pero a efectos prácticos, en la medida en que no se las pretende activar políticamente, más que como divisorias, en el sentido de Lipset y Rokkan, habría que entenderlas como variables.

Una muestra reciente del callejón sin salida al que conduce seguir hablando de cleavages es el intento de Kriesi (1998) de combinar el enfoque de las divisorias sociales con la tesis de los nuevos valores posmaterialistas, tratando de mostrar la existencia de una divisoria estructural, dentro de las clases medias urbanas, de la que se derivaría un cleavage en términos de valores. Que la oportunidad fuera la Stein Rokkan Lecture de 1997 puede ser una buena justificación del intento, pero los resultados no son necesariamente verosímiles. Una cosa es hablar de divisorias de género o de raza (Brooks y Manza, 1997), para analizar las correlaciones entre tales categorías sociales y la conducta electoral, y otra pretender buscar una raíz estructural de las diferencias en valores. Si vamos a acabar encontrando un cleavage en cualquier diferencia social, mejor dejar de usar el término.

\section{Intereses y preferencias}

La aproximación teórica más usual al comportamiento electoral toma como punto de partida el enfoque de Downs (1957), y supone que los electores votan al partido que en su programa se aproxima más a sus propias preferencias. El modelo se complica cuando, en conformidad con las exigencias de la teoría de la decisión racional, el elector debe valorar también no sólo las promesas sino la probabilidad de que el partido en cuestión las cumpla, pues se debe considerar la capacidad de los candidatos y su probabilidad de ganar. Por mucho que se sienta atraído por su programa, un elector racional no debería votar a un partido cuyo candidato tiene muy escasas posibilidades de salir elegido o es sospechoso de incompetencia. 
Para mayor complicación, el elector debe valorar si el esfuerzo de buscar la información necesaria para decidir, más la mayor o menor molestia que supone el hecho en sí de votar, se verán compensados por la posible repercusión de su voto en el resultado final. Muchos de estos problemas se pueden obviar si se supone que entre los electores y los partidos a los que votan existe una relación de identificación. En primer lugar, esta relación les dota de una regla de actuación en el sentido de la racionalidad limitada (Simon, 1955), es decir, les permite tomar una opción satisfactoria, a la luz de la experiencia pasada, en términos de programa y candidatos. En segundo lugar, la reafirmación de esta relación de identificación les proporciona un incentivo expresivo para votar (Riker y Ordeshook, 1968; Fiorina, 1976).

Ahora bien, como ya se apuntaba antes, la identificación partidaria ha disminuido numéricamente en el conjunto del electorado y su intensidad es menor. Parece indiscutible que muchos electores no deciden su voto en términos de una identificación previa, y que otros, a los que podemos considerar inicialmente identificados, deciden abstenerse o cambiar su identificación, a menudo tras un momento de abstención. Es necesario plantearse por tanto el problema de la decisión de votar como un problema de elección racional, y no es posible eludirlo explicando el voto a un partido como fruto de un simple proceso de socialización política que se vería reforzado si los resultados del voto son satisfactorios. Hay momentos en que los electores identificados consideran insatisfactorio el resultado de su voto, y en cada proceso electoral hay votantes que deciden deliberadamente el sentido de su voto.

Esto, a su vez, nos lleva a plantearnos la cuestión de las preferencias de los electores. En primer lugar, si éstas son arbitrarias o, como cabe sospechar, guardan alguna relación con la posición individual o familiar en la estructura social. En segundo lugar, la forma en que a partir de estas preferencias deciden el sentido de su voto. Esta segunda cuestión es más compleja de lo que parece porque sólo admite soluciones unívocas si suponemos que las preferencias son agregables a lo largo de un único eje sobre el que la oferta de los partidos representa puntos discretos, o bien que cada vez se vota sobre una sola cuestión y que los electores tienen preferencias separables (Enelow y Hinich, 1984: 22). Sobre esta base se presenta normalmente la teoría espacial del voto, y es comprensible que así sea, porque si consideramos $n$ ejes de preferencias nos surge inmediatamente el problema de los ciclos de votación (la paradoja de Condorcet o teorema de imposibilidad de Arrow), es decir, el problema de la inestabilidad de las mayorías.

Como es evidente, sin embargo, en un proceso electoral los votantes eligen gobernantes o representantes en función de sus propuestas sobre $n$ cuestiones, y las preferencias no son separables. Ahora bien, el problema teórico no es tan inmanejable como se le suele considerar: cuando dos candidatos compiten en un espacio $n$-dimensional de preferencias, existe un conjunto de puntos (el uncovered set) que representan propuestas de política que no pueden ser derrotadas, y que constituye algo así como la región central de las preferencias de los votantes (Ordeshook, 1986: 180-187; McKelvey, 1986). Imponiendo condiciones razonablemente restrictivas, este conjunto representa un núcleo de políticas del que no puede desviarse sustancialmente la oferta de los partidos, y ofrece por tanto una versión realista del teorema del votante medio (median voter) de Black (1958), sobre la convergencia de la oferta política de los partidos con la mediana de las preferencias de los votantes en un único eje de preferencias.

La importancia de esta cuestión se deriva de que si no existe esta convergencia carece de base la propia teoría democrática, ya que podrían formarse mayorías que no respondieran sustancialmente a las preferencias de los electores. Y la necesidad de plantearse la generalización del teorema de Black a $n$ dimensiones surge de los estudios empíricos que muestran la existencia de un número creciente de votantes que deciden su voto en función 
de cuestiones concretas (issues) y no necesariamente a partir de la posición global de los partidos en un único eje posicional (ideológico, por ejemplo). En particular, parece evidente que la simple autoidentificación ideológica no explica el sentido del voto, más allá de cualquier otro factor.

La idea de que los ciudadanos deciden su voto en función de cuestiones concretas choca con otra perspectiva muy extendida, que supone que el bienestar material es la cuestión dominante a la hora de decidir el voto, y que el estado y perspectivas de la economía son el factor decisivo del comportamiento electoral. En su forma más simple, como ilustración de la teoría de la elección racional aplicada a la decisión de votar, se supone que el elector busca maximizar su propio bienestar material, y que decide su voto según las perspectivas económicas que para sus intereses ofrecen los diferentes programas partidarios, con las consabidas complicaciones de valorar la capacidad de los respectivos candidatos, la verosimilitud de sus promesas y las posibilidades reales que tienen de ganar.

Este planteamiento, en general, es el que podríamos llamar del voto económico. Introduce ante todo la idea, muy coherente con el egoísmo metodológico de la teoría de la elección racional, de que al elector sólo le preocupa el estado de su propio bolsillo, y en segundo lugar la de que el potencial votante valora las perspectivas futuras de la economía a la hora de decidir su voto. Sin embargo, un modelo tan sencillo ofrece serias dificultades a la hora de explicar el voto, en particular la dificultad de valorar la sinceridad y los efectos posibles de las promesas electorales. Ciertamente, en la mayor parte de los países se ha producido una familiaridad creciente de los electores informados con las complejidades de la economía, pero aun así no es tan fácil saber si un programa será llevado realmente a la práctica, si funcionará como se espera, y si sus efectos imprevistos no anularán sus ventajas esperadas.

Para eludir esta dificultad la teoría del voto económico adoptó la perspectiva del voto retrospectivo (Fiorina, 1978; Lewis-Beck, 1988), según la cual los electores juzgan al gobierno anterior por sus resultados y le castigan votando a la oposición o le premian revalidando su mandato. Pero desde esta perspectiva ha surgido una variante significativa: si se controla la identificación partidaria, aparece que lo que influye en el elector es su percepción del estado general de la economía, no el estado de su propio bolsillo. El voto económico sería un voto sociotrópico, no egotrópico (Kinder y Kiewiet, 1979). Cabe interpretar este hecho como una forma de valoración de la competencia de los gobernantes antes que de sus programas, lo que, si bien es racional, no corresponde a lo inicialmente esperable en la teoría.

Posteriormente, sin embargo, los estudios acumulados parecen mostrar que el estado de la economía no decide necesariamente el voto: se trataría sólo de un factor de influencia contingente, y que debería analizarse dentro de un contexto político más amplio (Leithner, 1993). Por poner un ejemplo evidente, una mala situación económica puede llevar a los ciudadanos a votar a la oposición sólo si la actuación de ésta ofrece cierta previsibilidad y garantías de competencia. Si el riesgo es demasiado alto, por el contrario, pueden optar por lo malo conocido. Las variables específicamente políticas determinan la interpretación que los ciudadanos hacen de los hechos económicos, y entre esas variables se incluye la reputación de los distintos partidos, los niveles de identificación con ellos, o la capacidad de los gobernantes para atribuir convincentemente a factores ajenos los males de la economía.

La tentación de mantener, sin embargo, que las cuestiones relacionadas con el bienestar material son las que deciden el sentido del voto, resulta muy grande, y probablemente mayor a consecuencia del famoso cartel en la oficina electoral de Clinton en 1992: "It's the economy, stupid". La frase feliz revela bastante bien por qué perdió Bush, pero no explica 
necesariamente por qué ganó Clinton. El análisis de la campaña de 1992 parece revelar en cambio qué cuestiones concretas, a las que aparecía vinculado el candidato demócrata, le dieron la victoria: la libertad de elegir (en la interrupción del embarazo) y su propuesta de universalizar la asistencia sanitaria, entre otras (Wattenberg, 1995).

Supongamos entonces que la economía es sólo una de las cuestiones, o conjuntos de cuestiones, sobre las que los ciudadanos deciden su voto, o bien un parámetro que interviene en su valoración de estas cuestiones. El problema que se presenta es saber si las preferencias de los electores sobre estas cuestiones son o no agregables sobre un único eje. Inglehart $(1977,1990)$ viene sosteniendo que desde los años 70 las preferencias de los electores se agrupan a lo largo de dos ejes, uno tradicional, relacionado con el bienestar material, y otro de nuevos valores posmaterialistas, relacionado con valores como la autonomía individual y la calidad de vida. En un horizonte tradicional, los intereses materiales del elector determinarían sus preferencias. Eso no impediría que también deseara conservar el medio ambiente, o que valorara altamente su autonomía individual, pero aceptaría soluciones de compromiso entre los dos tipos de preferencias dando prioridad al bienestar material. Ahora este compromiso no sería aceptable por quienes mantienen los nuevos valores.

La crisis de los años 90, y el crecimiento del desempleo estructural en Europa, han llevado a muchos autores a dudar de la validez de la tesis de Inglehart: las preferencias tradicionales por el bienestar material serían nuevamente las dominantes en la vida política de nuestras sociedades. Pero esto sólo es cierto en parte: aunque las preocupaciones por el empleo y el ingreso sean las dominantes, coexisten con otras no reducibles (en el sentido de agregables) a ellas. El electorado está segmentado en términos de preferencias, y la mayoría potencial que apoyaría una política, según un determinado eje de preferencias, se disgregaría o reduciría a una minoría ante las consecuencias de esta política según otro eje de preferencias.

La hipótesis central, en este punto, es que la diferenciación social traída por el desarrollo económico ha llevado a las personas a moverse simultáneamente en varias situaciones sociales (en el trabajo, en el consumo, en el ocio, como residentes, como ciudadanos) y multiplica las identidades sociales posibles. Esta variedad situacional priva de un anclaje único las preferencias personales, y se traduce en un auge de identidades colectivas (culturales, lingüísticas, étnicas, organizaciones o movimientos de objetivo único). Ahora bien, las identidades colectivas deben entenderse como metapreferencias u ordenaciones de los ordenamientos de preferencias individuales (Sen, 1977; Hirschman, 1984), y su multiplicación equivale por tanto a la multiplicación de los ejes de preferencias.

Planteadas así las cosas, es inevitable pensar en la extensa discusión ya existente sobre la relación entre partidos políticos y movimientos sociales. Pero este enfoque atañe también a otra cuestión más general: la autoidentificación de los electores como factor decisivo para la interpretación de sus propios intereses. Si un ciudadano puede adscribirse a más de una identidad social, la ordenación de sus preferencias, y por tanto la percepción de sus intereses, no serán algo determinado de antemano por su posición en la estructura social (por su posición de clase), sino algo que puede cambiar en función de factores políticos.

\section{Clase, preferencias y cuestiones}

En el horizonte tradicional, todos los aspectos de la vida social dependen de la posición de clase, y por tanto podemos deducir de ella los intereses de las personas. Si no actúan en coherencia con estos intereses (si manifiestan unas preferencias incoherentes) se presenta el problema de la falsa conciencia. Las anomalías que se presentan desde este 
planteamiento se pueden intentar afrontar por dos vías. La primera, en la que destaca el programa de investigación de E.O. Wright, es buscar definiciones de la posición de clase que expliquen mejor la existencia real de las personas que las tradicionales relaciones de producción marxistas o la posición en el mercado weberiana. No es evidente, sin embargo, que la mayor complicación del marco teórico se justifique en términos de mayor rendimiento empírico.

La segunda posibilidad es admitir que la posición estructural condiciona (o determina, si se quiere) los intereses materiales de las personas, pero no sus preferencias, y que las decisiones individuales se toman en función de los intereses percibidos. Estos serían el resultado de la interpretación de los intereses materiales en el marco individual de preferencias. Pero para no dejar demasiado margen a la arbitrariedad se puede suponer que las preferencias vienen también definidas por la posición estructural, y que la interpretación individual se refiere únicamente a su ordenación, a lo que hemos llamado metapreferencias. (Al hablar de preferencias lo hacemos en el sentido de la elección racional, como un conjunto transitivo y ordenado.)

En este marco, los procesos de autoidentificación social y política afectan a las metapreferencias individuales, y éstas serían las que decidirían el voto. Las identificaciones políticas previas afectarían de forma distinta, según su fuerza, a las metapreferencias individuales: las identificaciones fuertes (incondicionales, por decirlo así) llevarían a aceptar como válido, como adecuado para los propios intereses el ordenamiento de objetivos introducido en la propuesta partidaria, mientras que las débiles llevarían sólo a sobreestimar los aspectos positivos y descontar parcialmente los negativos.

La clave del problema reside en las identidades sociales: las identificaciones políticas sólo se mantienen en la medida en que son compatibles con la identidad personal, entendiendo ésta como resultado de la adscripción a una o varias identidades colectivas (familiares, laborales, de tiempo libre, asociativas, etc.) No hace falta tomarse al pie de la letra la teoría de la disonancia cognoscitiva para aceptar que si todos los entornos de una persona rechazan su identidad política, aquélla deberá modificar su identidad o sus entornos (las rupturas familiares en situaciones de crispación política son un hecho bastante documentable).

¿Cuál es el sentido de las campañas políticas? Debemos partir del hecho evidente de que éstas no se proponen usualmente activar divisorias preexistentes (con la conocida excepción de los nacionalismos, y con consecuencias también conocidas), y sin embargo parece razonable creer que su objetivo es movilizar a unos grupos electorales determinados en función de sus intereses. La idea que cabe proponer es que el sentido de una campaña es ajustar las preferencias de estos grupos a una metaordenación básicamente compatible con el programa partidario. Activar una divisoria excluye identidades sociales que no entran en ella (los profesionales y los trabajadores de los servicios, por ejemplo, en el caso de un discurso obrerista). En cambio es posible influir en la ordenación de las preferencias de los trabajadores manuales, para hacerlas coincidir con la oferta partidaria, en función de cuestiones concretas, que no necesariamente provoquen el rechazo de otros grupos.

En la perspectiva clásica, tales cuestiones vendrían determinadas por los intereses y demandas de los electores y por las políticas posibles según el entorno. En teoría cabe dar prioridad a los intereses (definidos en términos estructurales) o a la autonomía de los partidos y gobiernos para diseñar sus políticas. En el caso español, Boix (1977) ha hecho hincapié en las restricciones que el entorno introduce a partir de una elección estratégica de política, y González (1996) ha relacionado los cambios en los apoyos electorales de los 
socialistas con las consecuencias no deseadas de tal estrategia y de las posteriores restricciones. Resumiendo (demasiado), la estrategia de inversión en capital humano e infraestructuras, más las consecuencias del paquete redistributivo de 1989, en las nuevas condiciones creadas por la recesión europea de 1991-92, habrían tenido consecuencias inevitables en términos económicos (explosión del paro) y electorales.

En la perspectiva que aquí se apunta, ni el momento del paquete redistributivo ni los cambios en los apoyos electorales del PSOE pueden entenderse sin introducir factores políticos. La huelga general de 1988 no se puede explicar únicamente en función de la política económica de los gobiernos socialistas, sino que cobra sentido en el marco de las relaciones entre las élites socialistas y las sindicales, y esto incluye a su vez las relaciones de éstas con IU. Es preciso comprender este juego de cuatro actores para explicar la huelga general del 14-D y la posterior aprobación del paquete redistributivo sin acuerdo con los sindicatos. El gobierno pretendía evitar que la cuestión redistributiva (la deuda social) se convirtiera en bandera de la oposición, una vez que la confrontación con los sindicatos impedía convertirla electoralmente en cuestión de gobierno.

La desavenencia con los sindicatos también es un factor explicativo fundamental en la evolución de los apoyos electorales de los socialistas. Tanto en las elecciones de 1993 como en las de 1996, las críticas sindicales debilitaban la confianza que el PSOE exigía a los electores en una confrontación en la que no existían medidas estelares ni grandes cuestiones que pudieran movilizar a los electores que se autoidentificaban como trabajadores. Pero el otro factor, dada la situación económica de crisis y alto desempleo, fue la vinculación que la oposición logró establecer entre problemas económicos y continuidad del gobierno socialista.

La fórmula "paro, corrupción y despilfarro" no sólo apuntaba tres flancos de crítica al gobierno: los asociaba en una relación de causa y efecto. El paro era consecuencia de una mala gestión en la que los socialistas sólo se habían ocupado de enriquecerse o de beneficiar a sus amigos, dilapidando en subsidios, empleos productivos y obras faraónicas los recursos públicos. Incluso quienes no aceptaban esta idea eran permeables a la de que la situación económica y el empleo no podían mejorar mientras permaneciera el gobierno socialista, y con él una insufrible crispación política que no permitía, ni a los gobernantes ni a los ciudadanos, plantearse en serio las decisiones sobre el futuro de la economía española.

Da lo mismo, a efectos del análisis, cuáles fueran los medios para lograr que esa fórmula calara en la opinión pública, como no importa demasiado su mayor o menor correspondencia con los hechos reales. La oposición consiguió que la cuestión central de las elecciones de 1996 fuera sacar a los socialistas del gobierno, y que sectores sociales muy significativos aceptaran que, sin un cambio de gobierno, la situación del país y su propia situación personal no podían mejorar: que el cambio de gobierno fuera el eje de sus metapreferencias. (Podemos incluso incluir en este grupo a los electores sofisticados, que anticiparan la reacción de los mercados a uno u otro resultado electoral.)

Por su lado, la campaña socialista sólo pudo acentuar los riesgos implícitos en el cambio de gobierno y reforzar la identificación de sus electores subrayando la parcialidad y la intolerante agresividad de sus adversarios. Que los sectores más identificados fueran los que más se venían beneficiando directamente de las medidas redistributivas del gobierno socialista, y los que menos confiaban en los medios para formar su opinión, resulta tan esperable como en el marco clásico, pero, a diferencia de aquél, no constituye toda la historia.

El ejemplo español de 1996 muestra que la autonomía de los actores políticos no puede 
sobreestimarse, pero no necesariamente porque las restricciones económicas y la estructura social les condicionen de forma casi absoluta, sino porque los factores políticos no son simplemente discursivos. A menudo exigen tiempo para ser modificados, y sin un cambio en determinadas condiciones del entorno político no es posible introducir en la agenda, con verosimilitud, las cuestiones que más podrían beneficiar a un actor. El esfuerzo de los socialistas, desde 1993, por enviar señales al público de su voluntad real de abrir una época nueva, por ejemplo, no pudo cuajar ante los conflictos internos que impidieron dar, en el gobierno y en el partido, una imagen verosímil de renovación.

La misma persecución de los hechos de corrupción de la época anterior, de hecho, no benefició a los socialistas entre 1993 y 1996. La cuestión de la corrupción era una cuestión propia de la oposición: cuanto mas a menudo aparecía en los medios, aunque fuera vinculada a la actuación del gobierno socialista en su persecución, más reforzaba la metapreferencia por el cambio de gobierno, y por tanto la estrategia de la oposición (Kleinnijenhuis y De Ridder, 1998). Es difícil imaginar qué estrategia, discursiva, política o económica, podría haber evitado que los socialistas llegaran como perdedores a las elecciones de 1996, incluso si la recuperación de la economía hubiera sido percibida por los electores.

A fin de cuentas, entonces, lo que deberíamos discutir no es la relación entre clase y voto, sino los límites de las explicaciones económicas del voto, sociotrópicas o egotrópicas, retrospectivas o prospectivas. Si nos tomamos en serio la existencia de factores políticos que influyen en la decisión de votar y en el sentido del voto, podemos tomarnos en serio las estrategias políticas y las campañas electorales, mientras que si mantenemos la explicación puramente económica el único trabajo serio sería diseñar la estrategia económica de los gobiernos. En la primera perspectiva, conocer la estructura social es un requisito para hacer política, en la segunda una actividad subordinada a la de los economistas. Ni siquiera como horizonte gremial parece muy atractivo.

\section{Referencias}

Abramson, P.R. (1976), "Generational change and the decline of party identification in America, 1952-1974", American Political Science Review 70: 469-478.

Abramson, P.R. (1992), "Of time and partisan instability in Britain", British Journal of Political Science 22: 381395.

Arrow, K.J. (1951), Elección social y valores individuales, Madrid: Instituto de Estudios Fiscales, 1974 [Social choices and individual values, $2^{a}$ ed., New Haven: Yale University Press, 1963].

Bartolini, S., y Mair, P. (1990), Identity, competition, and electoral availability: the stabilization of European electorates, 1885-1985, Cambridge: Cambridge University Press.

Black, D. (1958), The theory of committees and elections, Cambridge: Cambridge University Press.

Boix, C. (1997), "Dos décadas de política económica española", Claves 77: 34-42.

Brooks, C., y Manza, J. (1997b), "Social cleavages and political alignments: US presidential elections, 1960 to 1992", American Sociological Review 62: 937-946.

Burnham, W.D. (1970), Critical elections and the mainsprings of American politics, Nueva York: Norton. 
Converse, P.E. (1969), "Of time and partisan stability", Comparative Political Studies 2: 139-171.

Downs, A. (1957), An economic theory of democracy, Nueva York: Harper \& Row [Teoría económica de la democracia, Madrid: Aguilar, 1973].

Enelow, J.E., y Hinich, M.J. (1984), The spatial theory of voting: an introduction, Cambridge: Cambridge University Press.

Fiorina, M.P. (1976), "The voting decision: instrumental and expressive aspects", Journal of Politics 38: 390413.

Fiorina, M.P. (1978), "Economic retrospective voting in American national elections: a microanalysis", American Journal of Political Science 22: 426-443.

Franklin, M. (1992), "The decline of cleavage politics", en M. Franklin, T. Mackie, H. Valen et al., Electoral change: responses to evolving social and attitudinal structures in Western countries, 383-405, Cambridge: Cambridge University Press.

González, J.J. (1996), "Clases, ciudadanos y clases de ciudadanos: el ciclo electoral del pos-socialismo", Revista Española de Investigaciones Sociológicas 74: 45-76.

Green, D.P., y Palmquist, B. (1994), "How stable is party identification?", Political Behavior 43: 437-466.

Hirschman, A.O. (1984), "Contra la parquedad: tres maneras fáciles de complicar algunas categorías del discurso económico", en Enfoques alternativos sobre la sociedad de mercado, 142-159, México: Fondo de Cultura Económica, 1989 ["Against parsimony: three easy ways of complicating some categories of economic discourse", American Economic Review 74 (2): Papers and proceedings, 88-96].

Inglehart, R. (1977), The silent revolution: changing values and political styles among Western publics, Princeton: Princeton University Press.

Inglehart, R. (1990), El cambio cultural en las sociedades industriales avanzadas, Madrid: Centro de Investigaciones Sociológicas, 1991 [Culture shift in advanced industrial society, Princeton: Princeton University Press].

Key, V.O. (1955), "A theory of critical elections", Journal of Politics 17: 3-18.

Kinder, D.R., y Kiewiet, D.R. (1979), "Economic discontent and political behavior: the role of personal grievances and collective economic judgments in congressional voting", American Journal of Political Science 23: $495-527$.

Kleinnijenhuis, J., y De Ridder, J.A. (1998), "Issue news and electoral volatility: a comparative analysis of media effects during the 1994 election campaigns in Germany and the Netherlands", European Journal of Political Research 33: 413-437.

Kriesi, H. (1998), "The transformation of cleavage politics", European Journal of Political Research 33: 165185.

Leithner, C. (1993), "Economic conditions and the vote: a contingent rather than a categorical influence", British Journal of Political Science 23: 339-372.

Leithner, C. (1997), "Of time and partisan stability revisited: Australia and New Zealand 1905-90", American Journal of Political Science 41: 1104-1127.

Lewis-Beck, M.S. (1988), Economics and elections: the major Western democracies, $2^{\mathrm{a}}$ ed., Ann Arbor: University of Michigan Press, 1990.

Lipset, S.M., y Rokkan, S. (1967), "Cleavage structures, party systems, and voter alignments", en S.M. Lipset y S. Rokkan, comps., Party systems and voter alignments: cross national perspectives, 1-67, Nueva York:

Free Press ["Estructuras de división, sistemas de partidos y alineamientos electorales", en G.A. Almond et al., 
Diez textos básicos de ciencia política, 231-273, Barcelona: Ariel, 1992].

Manza, J., Hout, M., y Brooks, C. (1995), "Class voting in capitalist democracies since World War II: dealignment, realignment, or trendless fluctuation?", Annual Review of Sociology 21: 137-162.

McKelvey, R.D. (1986), "Covering, dominance, and the institution-free properties of social choice", American Journal of Political Science 30: 283-314.

Ordeshook, P.C. (1986), Game theory and political theory: an introduction, Cambridge: Cambridge University Press.

Riker, W.H., y Ordeshook, P.C. (1968), "A theory of the calculus of voting", American Political Science Review 62: 25-42 ["Una teoría del cálculo de votar", en J.M. Colomer, comp., Lecturas de teoría política positiva, 299338, Madrid: Instituto de Estudios Fiscales, 1991].

Schickler, E., y Green, D.P. (1997), "The stability of party identification in western democracies: results from eight panel surveys", Comparative Political Studies 30: 450-483.

Sen, A. (1977), "Los tontos racionales: una crítica de los fundamentos conductistas de la teoría económica", en F. Hahn y M. Hollis, comps., Filosofía y teoría económica, 172-217, México: Fondo de Cultura Económica, 1986 ["Rational fools: a critique of the behavioral foundations of economic theory", Philosophy and Public Affairs 6: 317-344].

Shively, W.P. (1972), "Party identification, party choice, and voting stability: the Weimar case", American Political Science Review 66: 1203-1227.

Simon, H.A. (1955), "A behavioral model of rational choice", Quarterly Journal of Economics 69: 99-118 [reimpreso en Models of man, 241-260, Nueva York: Wiley, 1957].

Wattenberg, M.P. (1995), "Why Clinton won and Dukakis lost: an analysis of the candidate-centered nature of American party politics", Party Politics 1: 245-260.

Ponencia para la sesión conjunta "Clase, política y ciudadanía" de los grupos de Sociología Política y de Desigualdad y Clases Sociales, VI Congreso Español de Sociología, A Coruña, 24-26 de septiembre de 1998. Este trabajo forma parte del proyecto Actores sociales e instituciones políticas en los procesos de reforma económica (SEC96-0639). Publicado en Revista Española de Investigaciones Sociológicas 90: 8195, 2000. 